\title{
STABILITY OF RATIONAL MULTISTEP APPROXIMATIONS OF HOLOMORPHIC SEMIGROUPS
}

\author{
C. PALENCIA
}

\begin{abstract}
In this paper we prove the stability of semidiscretizations in time of holomorphic semigroups in Banach spaces by means of $A(\alpha)$-stable rational multistep methods. No assumptions on the method other than $\mathrm{A}(\alpha)$-stability are required. Our result is applicable in the maximum norm analysis of parabolic problems.
\end{abstract}

\section{INTRODUCTION}

The present paper is devoted to the study of the stability of rational multistep semidiscretizations in time of parabolic initial value problems. As is well known, a parabolic problem generates a dynamical system in a suitable underlying function space which, in general, is not a Hilbert space. For instance, Banach spaces of continuous functions with the maximum norm are common. In these cases, unless some kind of maximum principle is available, the analysis of the discretizations could be difficult. Our main result establishes the stability of such semidiscretizations under the natural condition of $\mathrm{A}(\alpha)$-stability of the method.

From an abstract point of view, we will say that a linear initial value problem

$$
\left\{\begin{array}{l}
u^{\prime}(t)=A u(t), \\
u(0)=u_{0} \in D(A)
\end{array}\right.
$$

is parabolic when $A: D(A) \subset X \rightarrow X$ is the infinitesimal generator of an analytic semigroup $e^{t A}, 0 \leq t<+\infty$, of linear and bounded operators in a Banach space $X$ (see e.g. [9] and [11]). Henceforth, we will restrict attention to the case of complex Banach spaces. The case of real Banach spaces is of practical interest but it can be handled easily by complexifying $X$ (see e.g. [20]). Given $\alpha \in(0, \pi / 2)$ and $M \geq 1$, we denote by $S(X, M, \alpha)$ the set formed by all linear, densely defined operators $A: D(A) \subset X \rightarrow X$ such that the spectrum of $A$ lies in the sector

$$
S_{\alpha}:=\{0\} \cup\{z \in C: z \neq 0,|\arg (-z)| \leq \alpha\}
$$

and

$$
\left\|(z I-A)^{-1}\right\| \leq M /|z|, \quad z \in C, z \notin S_{\alpha} .
$$

Received by the editor March 2, 1993 and, in revised form, December 2, 1993 and March 23, 1994.

1991 Mathematics Subject Classification. Primary 65J10, 65M12.

Key words and phrases. Parabolic problems, holomorphic semigroups, analytic semigroups, multistep and rational methods, stability, $\mathrm{A}(\alpha)$-stability, maximum norm, Banach spaces. 
Operators in $S(X, M, \alpha)$ are referred to as sectorial (see e.g. [6]). We have (see e.g. $[6,11])$ that problem (1) is parabolic if, and only if, for some $\alpha \in(0, \pi / 2)$, $M \geq 1$ and $\mu \in R$ the shifted operator $(A-\mu I)$ belongs to $S(X, M, \alpha)$. We point out that $A \in S(X, M, \alpha)$ implies that $e^{t A}, 0 \leq t<+\infty$, is a bounded semigroup.

Let $k \geq 1$ be an integer. A rational $k$-step method for (1) (see e.g. [2]) approximates the value $e^{t A} u_{0}, t=n h>0, h>0, n$ integer, $u_{0} \in X$, of a generalized solution of (1) by the solution $u_{n}$ of a recurrence

$$
u_{j}=f_{0}(h A) u_{j-k}+f_{1}(h A) u_{j-k+1}+\cdots+f_{k-1}(h A) u_{j-1}, \quad j \geq k,
$$

where $f_{0}(z), f_{1}(z), \ldots, f_{k-1}(z)$ are rational functions that define the method. When $k \geq 2$, the starting values $u_{1}, \ldots, u_{k-1}$ are also needed, and we assume that they are provided by suitable auxiliary procedures. Furthermore, we suppose that the rational functions $f_{0}(z), f_{1}(z), \ldots, f_{k-1}(z)$ are bounded on the spectrum of $h A$. Thus, (2) makes sense for arbitrary starting values in $X$. Examples of rational $k$-step methods are rational one-step methods (see e.g. [1, 10]), linear multistep methods (see e.g. [4, 13]) and general multistep methods (see e.g. [8]). The amplification matrix of the method is given by

$$
F(z)=\left[\begin{array}{cccc}
f_{k-1}(z) & \cdots & f_{1}(z) & f_{0}(z) \\
1 & \cdots & 0 & 0 \\
\cdots & \cdots & \cdots & \cdots \\
0 & \cdots & 1 & 0
\end{array}\right]
$$

and the recurrence (2) is equivalent to

$$
U_{j}=F(h A) U_{j-1}, \quad j \geq k,
$$

where $U_{j}=\left[u_{j}, u_{j-1}, \ldots, u_{j-k+1}\right]^{T} \in X^{k}, j \geq k-1$. The Lax stability (see e.g. [19] and [21]) of the method, when applied to problem (1), requires that for each $T>0$ there exists a constant $C>0$ such that

$$
\left\|F(h A)^{n}\right\| \leq C, \quad h>0, n h \leq T .
$$

Here the operator norm of $F(h A)^{n}: X^{k} \rightarrow X^{k}$ is measured with respect to any norm in $X^{k}$ generating the standard topology.

Let us recall some basic notions. The stability region $S R$ of a rational $k$-step method with amplification matrix $F(z)$ is defined as

$$
S R=\{z \in C \cup\{\infty\}: F(z) \text { is power bounded }\} .
$$

It is easy to see, because $F(z)$ is a Frobenius matrix, that $z$ belongs to $S R$ if, and only if, the characteristic polynomial of the matrix $F(z)$ satisfies the root condition (see e.g. [7]), i.e., its roots lie in the closed unit disk and roots with unit modulus are simple. Denote by $M_{k \times k}$ the set formed by all $k \times k$ complex matrices. For $F \in M_{k \times k}$ we denote by $\rho(F)$ the spectral radius of $F$. The method is said to be $\mathrm{A}(\alpha)$-stable, $0<\alpha \leq \pi / 2$, when $\{\infty\} \cup S_{\alpha} \subset S R$. The method is called strongly $\mathrm{A}(\alpha)$-stable when it is $\mathrm{A}(\alpha)$-stable and $\rho(F(\infty))<1$. Finally, when $\alpha=\pi / 2$, the terms A-stable and strongly A-stable are used.

Now we can state the following general stability theorem, which constitutes the main contribution of the present paper. 
Theorem 1. Let $\alpha \in(0, \pi / 2)$ and $M \geq 1$. Suppose that a rational $k$-step method with amplification matrix $F(z)$ is $\mathrm{A}(\alpha)$-stable. Then there exists a constant $C=C(F, \alpha, M)$ such that the inequality

$$
\left\|F(h A)^{n}\right\| \leq C, \quad h>0, n \geq 1,
$$

holds, for each complex Banach space $X$ and each $A \in S(X, M, \alpha)$.

We observe that the conclusion of Theorem 1 is stronger than Lax stability. In fact, this theorem shows the important qualitative feature that the method, as does problem (1) itself, generates bounded orbits, $U_{k-1}, U_{k}, \ldots$.

Next we comment briefly on several known results that are of interest in relation with Theorem 1 .

(a) Lax stability does not hold for general semigroups and A-stable rational $k$-step methods (see $[1,3]$ and [10]). A-stable linear multistep methods and semigroups of contractions in Hilbert spaces are considered, for instance, in [4, 13] and [18].

(b) The convergence of some strongly $\mathrm{A}(\alpha)$-stable rational $k$-step methods for holomorphic semigroups has been studied in [2] and [13]. However, when $k \geq 2$, these papers do not show the stability of the methods. In [2] and [13] the starting values are of the form $u_{j}=g_{j}(h A), 1 \leq j \leq(k-1)$, for some suitable auxiliary rational functions $g_{1}(z), \ldots, g_{k-1}(z)$. Then, because of convergence, [2] and [13] imply that the sequence $F(h A)^{n-k}\left[I, g_{1}(h A), \ldots, g_{k-1}(h A)\right]^{T}$, $n \geq k$, is bounded, but this is weaker than the stability estimate (3).

Concerning the stability of $\mathrm{A}(\alpha)$-stable methods and parabolic problems (1), with $A$ sectorial of the same angle $\alpha \in(0, \pi / 2)$, we have also:

(c) Lax stability holds for strongly $\mathrm{A}(\alpha)$-stable one-step methods (see $[1,12]$ and [14]). In [16] even variable stepsizes are allowed. [14]).

(d) Strongly $\mathrm{A}(\alpha)$-stable irreducible linear multistep methods are stable (see

(e) Stability was proved for general (not necessarily strongly) $\mathbf{A}(\alpha)$-stable methods in [3] and [15]. When restricted to one-step methods, our present proof is somewhat simpler than those of [3] and [15].

(f) More recently, in [17], stability has been established for a wide range of $\mathbf{A}(\alpha)$-stable rational multistep methods including, among other, the cases mentioned above. The applicability of [17] is somewhat restricted by the fact that a certain behavior of the spectral radius of the amplification matrix at 0 and at $\infty$ is required.

In summary, Theorem 1, in contrast with the results just reported, establishes the stability without extra assumptions about the $\mathrm{A}(\alpha)$-stable method. On the other hand, a rational $k$-step method that is Lax stable, when applied to any parabolic initial value problem (1) with $A$ sectorial of angle $\alpha \in(0, \pi / 2)$, is necessarily $\mathrm{A}(\alpha)$-stable. Therefore, the hypotheses in Theorem 1 are optimal.

The rest of the paper is as follows. In $\S 2$ we prove Theorem 1 . In $\S 3$ we state the extension of Theorem 1 to the case where the holomorphic semigroup is not bounded. Furthermore, we consider conditions guaranteeing a strictly contractive behavior. Finally, we give several convergence results.

\section{Proof of the Main Result}

In the sequel, $D_{z, r}$ denotes the closed disk in $C$ of center $z \in C$ and radius $r>0$. For $z=\infty$, we denote also 


$$
D_{\infty, r}=\{\infty\} \cup\left\{z \in C:|z| \geq r^{-1}\right\} .
$$

For $G=\left\{g_{l m}\right\}_{l, m=1}^{k} \in M_{k \times k}$ and a linear and continuous operator $B$ in $X$, we denote by $G \otimes B$ (Kronecker's product) the linear and continuous transformation in $X^{k}$ defined by the operator matrix $\left\{g_{l m} B\right\}_{l, m=1}^{k}$. We assume that norms in $M_{k \times k}$ and in $X^{k}$ are taken in such a way that

$$
\|G \otimes B\| \leq\|G\|\|B\| \quad \text { o }
$$

is satisfied, for each matrix $G \in M_{k \times k}$ and each linear and bounded operator $B$ in $X$.

We begin the proof with a discussion concerning the amplification matrix $F(z)$. Denote by $\Omega$ the complement in the Riemann sphere of the set formed by the poles of $F(z)$. Let $\zeta$ be either 0 or $\infty$ and set

$$
\Lambda_{\zeta}=\{\mu \in C: \operatorname{det}(\mu I-F(\zeta))=0 \text { and }|\mu|=1\} \text {. }
$$

Select $\delta>0$ satisfying $D_{\mu^{\prime}, \delta} \cap D_{\mu^{\prime \prime}, \delta}=\varnothing$ when $\mu^{\prime}, \mu^{\prime \prime} \in \Lambda_{\zeta}, \mu^{\prime} \neq \mu^{\prime \prime}$. Since the matrix $F(\zeta)$ satisfies the root condition, it is clear that any element of $\Lambda_{\zeta}$ is a simple characteristic root of $F(\zeta)$. This fact, together with the continuity of the characteristic roots of $F(z)$ with respect to $z \in \Omega$, shows that there exists $r \in(0,1)$ with $D_{\zeta, r} \subset \Omega$ and such that, for $z \in D_{\zeta, r}$ and $\mu \in \Lambda_{\zeta}$, the equation $\operatorname{det}(\lambda I-F(z))=0$ possesses exactly one root in each disk $D_{\mu, \delta}$. This root is denoted $\lambda_{\zeta, \mu}(z)$. Moreover, after reducing, if necessary, the size of $r$, we can suppose that, for $z \in D_{\zeta, r}$ and $\mu \in \Lambda_{\zeta}$, we have $\left|\lambda_{\zeta, \mu}(z)-\mu\right|<\delta$.

Fix $\mu \in \Lambda_{\zeta}$. Under the present circumstances, it is clear that the expression $\lambda_{\zeta, \mu}(z)$ is a holomorphic function of $z \in D_{\zeta, r}$. Furthermore, we have (see e.g. [11, §1.5]) that the projection $P_{\zeta, \mu}(z)$ onto the eigenspace of $F(z)$ corresponding to the eigenvalue $\lambda_{\zeta, \mu}(z)$ is given by the Cauchy integral

$$
P_{\zeta, \mu}(z)=\frac{1}{2 \pi i} \int_{\partial D_{\mu, \delta}}(\sigma I-F(z))^{-1} d \sigma, \quad \mu \in \Lambda_{\zeta}, z \in D_{\zeta, r},
$$

where $\partial D_{\mu, \delta}$ stands for the positive boundary of $D_{\mu, \delta}$. Therefore, the mapping $P_{\zeta, \mu}: D_{\zeta, r} \rightarrow M_{k \times k}$ is holomorphic.

Finally, after reducing again, if necessary, the size of $r$, we can assume that, for $z \in D_{\zeta, r}$, the characteristic roots of $F(z)$ other than the ones given by $\lambda_{\zeta, \mu}(z), \mu \in \Lambda_{\zeta}$, have modulus strictly less than one. In other words, denoting

$$
P_{\zeta}(z)=\sum_{\mu \in \Lambda_{\zeta}} P_{\zeta, \mu}(z) \quad \text { and } \quad Q_{\zeta}(z)=I-P_{\zeta}(z), \quad z \in D_{\zeta, r},
$$

we can suppose that

$$
\rho\left(F(z) Q_{\zeta}(z)\right)<1, \quad z \in D_{\zeta, r} .
$$

It is understood that in the above discussion we have chosen the same values of $\delta$ and $r$ for both $\zeta=0$ and $\zeta=\infty$.

Next we fix $A \in S(X, M, \alpha)$. Notice that the operators $h A, h>0$, also belong to $S(X, M, \alpha)$, so that we can restrict the proof of (3) to compositions $F(A)^{n}$ involving only $A$.

It is possible to prove, by means of the Neumann series (see e.g. [6, Theorem 7.9.5]), that there exist $L \geq 1$ and $0<\beta<\alpha$, depending on $M$ and $\alpha$ 
but independent of $A$ and the Banach space $X$, such that $S(X, M, \alpha) \subset$ $S(X, L, \beta)$. Let $\Gamma$ be the positively oriented boundary of the region $D_{0, r} \cup$ $S_{\beta} \cup D_{\infty, r}$. For $n \geq 1$, we can write

$$
F(A)^{n}=F(\infty)^{n} \otimes I+\frac{1}{2 \pi i} \int_{\Gamma} F(z)^{n} \otimes(z I-A)^{-1} d z .
$$

This representation of $F(A)^{n}$ is proved by a direct application of the residue theorem. The term $F(\infty)^{n} \otimes I$ is bounded independently of $n \geq 1$, because $\infty$ belongs to the stability region $S R$. One advantage of using the nonstandard path of integration $\Gamma$ is that, in the integral in (6), 0 and $\infty$ appear in a symmetric way. In fact, $F(1 / z) \otimes\left(z^{-1} I-A\right)^{-1} d(1 / z)$ exhibits in $\Gamma \cap D_{0,1}$ the same behavior as $F(z) \otimes(z I-A)^{-1} d z$. Thus, by using the transformation $z \rightarrow 1 / z$, we can restrict our attention to the contribution to (6) from the part of $\Gamma$ lying in the unit disk $D_{0,1}$. This path is the union of $\gamma$, the part of $\Gamma$ on the boundary of $D_{0, r}$, and $\sigma$, the union of the two segments $\left[-e^{i \beta},-r e^{i \beta}\right]$ and $\left[-r e^{-i \beta},-e^{-i \beta}\right]$.

The matrix $F(z)$ is power bounded for each $z \in \sigma$. Hence it is uniformly power bounded (see e.g. [2, Lemma 2] or [5]), i.e., there exists $C_{1}=C_{1}(F)>0$ such that

$$
\left\|F\left(-s e^{ \pm i \beta}\right)^{n}\right\| \leq C_{1}, \quad n \geq 1, r \leq s \leq 1 .
$$

Therefore, the contribution to (6) due to the segments is bounded by

$$
\frac{1}{\pi} \int_{r}^{1} C_{1} L d s / s=\frac{C_{1} L|\ln r|}{\pi},
$$

which is independent of $n \geq 1$.

On the other hand, for $z \in \gamma \subset D_{0, r}$ we have, by (4), that

$$
F(z)^{n}=F(z)^{n} Q_{0}(z)+F(z)^{n} P_{0}(z)=\left(F(z) Q_{0}(z)\right)^{n}+F(z)^{n} P_{0}(z) .
$$

By (5), the matrix $F(z) Q_{0}(z)$ is power bounded for each $z \in \gamma$. Hence, again by the results in [2] or [5], there exists $C_{2}=C_{2}(F)>0$ such that

$$
\left\|\left(F(z) Q_{0}(z)\right)^{n}\right\| \leq C_{2}, \quad n \geq 1, z \in \gamma .
$$

Therefore, for $n \geq 1$,

$$
\begin{aligned}
& \left\|\frac{1}{2 \pi i} \int_{\gamma}\left(F(z) Q_{0}(z)\right)^{n} \otimes(z I-A)^{-1} d z\right\| \\
& \quad \leq \frac{L}{2 \pi} \int_{\gamma}\left\|\left(F(z) Q_{0}(z)\right)^{n}\right\||z|^{-1}|d z| \leq \frac{L C_{2}}{2 \pi} \int_{-(\pi-\beta)}^{+(\pi-\beta)} d \varphi \leq L C_{2} .
\end{aligned}
$$

The remaining contribution to the integral in (6) is dealt with as follows. For $n \geq 1$, we have

$$
\begin{aligned}
& \frac{1}{2 \pi i} \int_{\gamma} F(z)^{n} P_{0}(z) \otimes(z I-A)^{-1} d z \\
& \quad=\frac{1}{2 \pi i} \int_{\gamma} \sum_{\mu \in \Lambda_{0}} F(z)^{n} P_{0, \mu}(z) \otimes(z I-A)^{-1} d z \\
& \quad=\sum_{\mu \in \Lambda_{0}} \frac{1}{2 \pi i} \int_{\gamma} \lambda_{0, \mu}(z)^{n} P_{0, \mu}(z) \otimes(z I-A)^{-1} d z .
\end{aligned}
$$


We estimate separately each term in the last expression. To this end, the path of integration $\gamma$ is deformed according to both $n \geq 1$ and $\mu \in \Lambda_{0}$. In order to simplify the notation, in the rest of the proof we suppose that we fix $\mu \in \Lambda_{0}$ and then we set $\lambda(z):=\lambda_{0, \mu}(z)$, for $z \in D_{0, r}$, and $p:=\max \left\{\left\|P_{0, \mu}(z)\right\|: z \in D_{0, r}\right\}$. We have $|\lambda(z)| \leq 1$ for each $z \in S_{\alpha} \cap D_{0, r}$. The case when $\lambda$ is constant on $D_{0, r}$ is trivial, so that we will suppose that $\lambda$ is not constant. Consideration of the Taylor expansion of $\lambda(z)$ at the origin leads to

$$
\lambda(z)=\lambda(0)+a z^{m}+O\left(z^{m+1}\right),
$$

where $a \neq 0$ and $m \geq 1$. Furthermore, by the maximum principle for $\lambda(z)$, we have that $|\lambda(z)|<1$ for $z \in S_{\beta}, z \neq 0$. Moreover, it is straightforward to conclude (see also [3]) that there exists $c>0$ such that, for $z \in D_{0, r}$,

$$
|\lambda(z)| \leq \begin{cases}e^{-c|z|^{m}} & \text { if } z \in S_{\beta}, \\ e^{c|z|^{m}} & \text { if } z \notin S_{\beta} .\end{cases}
$$

For any integer $n \geq 1$ let $\gamma_{n}$ be the part of the positive boundary of $D_{0, r n^{-1 / m}}$ lying outside $S_{\beta}$. We deform $\gamma$ into the new path

$$
\gamma_{n}^{*}:=\left[-r n^{-1 / m} e^{-i \beta},-r e^{-i \beta}\right] \cup \gamma_{n} \cup\left[-r e^{i \beta},-r n^{-1 / m} e^{i \beta}\right] .
$$

The paths $\gamma$ and $\gamma_{n}^{*}$ share the same ends. Therefore, by virtue of (7), we have

$$
\begin{aligned}
& \left\|\frac{1}{2 \pi i} \int_{\gamma} \lambda_{0, \mu}(z)^{n} P_{0, \mu}(z) \otimes(z I-A)^{-1} d z\right\| \\
& \quad \leq \frac{L p}{2 \pi} \int_{\gamma_{n}^{*}}|\lambda(z)|^{n}|z|^{-1}|d z| \\
& \quad \leq \frac{L p}{\pi} \int_{r n^{-1 / m}}^{r} e^{-c n s^{m}} s^{-1} d s+\frac{L p}{2 \pi} \int_{-(\pi-\beta)}^{+(\pi-\beta)} e^{c n\left(r n^{-1 / m}\right)^{m}} d \varphi \\
& \quad \leq \frac{L p}{m \pi} \int_{r^{m}}^{+\infty} e^{-c u} u^{-1} d u+L p e^{c r^{m}},
\end{aligned}
$$

an expression that is independent of $n \geq 1$. This concludes the proof of the theorem.

\section{FURTHER EXTENSIONS}

In this final section we state several results which are directly related to Theorem 1. We suppose that the constants $\alpha \in(0, \pi / 2), M \geq 1$ and the complex Banach space $X$ are fixed. We consider a rational $k$-step method with amplification matrix $F(z)$ that is $\mathrm{A}(\alpha)$-stable.

First, let us comment on the case where, in (1), the generator satisfies $(A-$ $\mu I) \in S(X, M, \alpha)$ for some $\mu>0$. This case arises, for instance, in parabolic problems with either a source or a convective term. Then, there exist constants $C, \omega>0$ and $h_{0}>0$, depending only on $F, \alpha, M$ and $\mu$, such that the inequality

$$
\left\|F(h A)^{n}\right\| \leq C e^{\omega n h}, \quad 0<h<h_{0}, n \geq 1,
$$

holds.

Secondly, suppose that in (1) we have

$$
(A-\mu I) \in S(X, M, \alpha) \quad \text { for some } \mu<0 .
$$


This condition implies a contractive behavior of the form $\left\|e^{t A}\right\| \leq M^{\prime} e^{\mu t}, t \geq$ 0 . Now it is of interest to ascertain whether the numerical method exhibits the analogous property (8), for some $C>0, h_{0}>0$ and $\omega<0$. Let us suppose that there exist $\mu<0$ and $\omega<0$ such that the method satisfies (8), for any operator satisfying (9). Then, by applying this result to the test equation $u^{\prime}=(\mu+\lambda) u, \lambda \in S_{\alpha}$, it is easy to deduce both that the method is strongly $\mathrm{A}(\alpha)$-stable and that, for each $\beta \in(0, \alpha)$, there exist a pair of constants $\gamma>0$ and $r>0$ such that

$$
\rho(F(z)) \leq 1-\gamma|z|, \quad z \in S_{\beta} \cap D_{0, r} .
$$

The scope of (10) is studied in [17], where it is shown that this condition is fulfilled by a wide range of methods. This range includes, among other, $\mathrm{A}(\alpha)$ stable methods with $\alpha>\pi / 4$, irreducible $\mathrm{A}(\alpha)$-stable linear multistep methods and several general multistep methods. Here we state that, conversely, when the rational $k$-step method is strongly $\mathrm{A}(\alpha)$-stable and fulfills condition (10), there exist constants $C, \omega<0$ and $h_{0}>0$, depending only on $F, \alpha, M$ and $\mu<0$, such that (8) holds, for each operator $A$ in $X$ with $(A-\mu I) \in S(X, M, \alpha)$.

For the proofs of these results we apply a theorem analogous to Theorem 1 to matrices of the form $e^{-\omega h} F(h(z-\mu)), 0<h \leq h_{0}$, for a suitable choice of $\omega$ and $h_{0}$, and to the operators $(A+\mu I)$.

Finally, we study the convergence of the mothod under consideration. Denote

$$
E(z)=\left[e^{(k-1) z}, \ldots, e^{z}, 1\right]^{T},
$$

and assume that the method is consistent of order $p \geq 1, p$ integer, i.e.,

$$
F(z) E(z)=e^{z} E(z)+z^{p+1} G(z),
$$

where $G(z)$ is some vector-valued analytic function in $S_{\alpha}$.

Let $h>0, A \in S(X, M, \alpha)$ and an integer $n \geq k$ be fixed. Suppose that in (2) the starting values $u_{0}, u_{1}, \ldots, u_{k-1}$ are taken in such a way that $e^{j h A} u(0)-u_{j}=\varepsilon_{j} \in X, 0 \leq j \leq k-1$. Moreover, suppose that, at each step $j \geq k$, the recurrence (2) is solved with an error of $\varepsilon_{j} \in X$, i.e., we have

$$
u_{j}-f_{0}(h A) u_{j-k}-f_{1}(h A) u_{j-k+1}-\cdots-f_{k-1}(h A) u_{j-1}=\varepsilon_{j} .
$$

Then, by Theorem 1 , it is straightforward to see that there exists a constant $C_{s}>0$, depending only on the method, such that

$$
\left\|u\left(t_{n}\right)-u_{n}\right\| \leq C_{s} \sum_{j=0}^{n}\left\|\varepsilon_{j}\right\|+\left\|T_{n}(h A) u(0)\right\|,
$$

where the term $T_{n}(h A)$ accounts for the error produced by the method when neither starting errors nor source errors are present. Observe that the operator $T_{n}(h A)$ is defined, by means of the functional calculus, as the operator in $X$ given by the analytic function

$$
T_{n}(z)=[1,0, \ldots, 0] \cdot\left(F(z)^{n-k} E(z)-e^{(n-k) z} E(z)\right),
$$

evaluated in $h A$. Consideration of the telescopic identity

$$
\left(F(z)^{n-k} E(z)-e^{(n-k) z} E(z)\right)=\sum_{j=0}^{n-k-1} F(z)^{n-k-j-1}\left(F(z) E(z)-e^{z} E(z)\right) e^{j z}
$$


together with Theorem 1 shows readily that there exists $C_{e}>0$ such that, for smooth initial data $u(0) \in D\left(A^{p+1}\right)$, the estimate

$$
\left\|T_{n}(h A) u(0)\right\| \leq C_{e} t h^{p}\left\|A^{p+1} u(0)\right\|
$$

holds. If $(A-\mu I) \in S(X, M, \theta), \mu \in R$, an extra factor $e^{\omega t}$, with $\omega$ of the same sign of $\mu$, is added in the upper bound of the last inequalities (in the case $\mu<0$ we must assume that (10) holds).

On the other hand, suppose that the method fulfills (10). Then it is possible to prove (see [2, Theorem 7]), by means of an alternative integral representation for $T_{n}(h A) u(0)$, that there exist constants $C_{e, q}, 1 \leq q \leq p$, such that (cf. (12))

$$
\left\|T_{n}(h A) u(0)\right\| \leq C_{e, q} n^{-(p-q)} h^{q}\left\|A^{q} u(0)\right\|, \quad u(0) \in D\left(A^{q}\right) .
$$

In particular, for $q=p$ we get an $O\left(h^{p}\right)$ estimate as in (12), but for initial data in $D\left(A^{p}\right)$ instead of in $D\left(A^{p+1}\right)$. Furthermore, (13) clearly improves on (12) for large $t$.

Finally, we point out that, for strongly $\mathbf{A}(\alpha)$-stable methods fulfilling (10), the estimate (13) holds even for bad initial data, i.e., for $q=0$ (see [2, Theorem 8]).

\section{ACKNOWLEDGMENTS}

The author is most grateful to Dr. Garcia-Archilla and Professor Sanz-Serna, for several useful discussions, and to the anonymous referee for several detailed suggestions that have greatly contributed to improve the presentation of the material.

\section{BIBLIOGRAPHY}

1. P. Brenner and V. Thomée, On rational approximations of semigroups, SIAM J. Numer. Anal. 16 (1979), 683-694.

2. M. Crouzeix, On multistep approximation of semigroups in Banach spaces, J. Comput. Appl. Math. 20 (1987), 25-35.

3. M. Crouzeix, S. Larsson, S. Piskarev, and V. Thomée, The stability of rational approximations of analytic semigroups, BIT 33 (1993), 74-84.

4. M. Crouzeix and P.-A. Raviart, Approximation d'équations d'évolution linéaires par des méthodes multipas, Publ. Analyse Numérique et Mécanique, Université Rennes I, 1976.

5. G. Dahlquist, H. Mingyou, and R. LeVeque, On the uniform power-boundedness of a family of matrices and the applications to one-leg and linear multistep methods, Numer. Math. $\mathbf{4 2}$ (1983), 1-13.

6. H. O. Fattorini, The Cauchy problem, Addison-Wesley, Reading, MA, 1983.

7. E. Hairer, S. P. Nørsett, and G. Wanner, Solving ordinary differential equations I, SpringerVerlag, Berlin, 1987.

8. E. Hairer and G. Wanner, Solving ordinary differential equations II, Springer-Verlag, Berlin, 1991.

9. D. Henry, Geometric theory of semilinear parabolic equations, Lecture Notes in Math., vol. 840, Springer-Verlag, New York, 1981.

10. R. Hersh and T. Kato, High-accuracy stable difference schemes for well-posed initial value problems, SIAM J. Numer. Anal. 16 (1979), 670-682.

11. T. Kato, Perturbation theory for linear operators, 2nd ed., Springer-Verlag, New York, 1966.

12. S. Larsson, V. Thomée, and L. B. Wahlbin, Finite-element methods for a strongly damped wave equation, IMA J. Numer. Anal. 11 (1991), 115-142. 
13. M. N. Le Roux, Semidiscretization in time for parabolic problems, Math. Comp. 33 (1979), 919-931.

14. Ch. Lubich and $\mathrm{O}$. Nevanlinna, On resolvent conditions and stability estimates, BIT 31 (1991), 293-313.

15. C. Palencia, A stability result for sectorial operators in Banach spaces, SIAM J. Numer. Anal. 30 (1993), 1373-1384.

16. _ On the stability of variable stepsize rational methods for holomorphic semigroups, Math. Comp. 62 (1994), 93-103.

17. C. Palencia and B. Garcia-Archilla, Stability of multistep methods for sectorial operators in Banach spaces, Appl. Numer. Math. 12 (1993), 503-520.

18. P.-A. Raviart, Multistep method and parabolic equations, Université Paris VI, Laboratoire Analyse Numérique, L. A. 189, 1976.

19. R. D. Richtmyer and K. W. Morton, Difference methods for initial-value problems, 2nd ed., Wiley, New York, 1967.

20. A. F. Ruston, Fredholm theory in Banach spaces, Cambridge Univ. Press, Cambridge, 1986.

21. J. M. Sanz-Serna and C. Palencia, A general equivalence theorem in the theory of discretization methods, Math. Comp. 45 (1985), 143-152.

Departamento de Matemática Aplicada y Computación, Universidad de Valladolid, VAlladolid, SpaIN

E-mail address: palencia@cpd.uva.es 